\title{
Review on Theft Prevention System using Raspberry Pi and PIR Sensor
}

\author{
Sadhana Godbole \\ NBNSSOE
}

\author{
Shivani Deshpande \\ NBNSSOE
}

\author{
Neha Barve \\ NBNSSOE
}

\author{
Sakshi Galim \\ NBNSSOE
}

\begin{abstract}
In this proposed system, theft can be prevented using Raspberry pi and PIR sensors. In this system PIR sensors, camera, buzzer, relay, GSM module are connected to Raspberry pi. PIR sensors are able to detect the presence of human being. After detecting any person, relays are triggered to switch on the lights. As soon as lights are on, buzzer will start ringing for specified time. After lights are turned on an image will be captured using cameras. This captured image will immediately upload on the web-page, which can be used as evidence. In addition, victim receives a notification in the form of text message with help of GSM module. Power supply to this system is given by an adapter. In absence of electricity battery backup is also provided. As the lights turn on and the buzzer rings, neighborhood becomes aware about a theft. This will make the thief scared and thief would try to escape the location. Thief is not able to execute his/her plan, belongings will be safe. This system can be implemented in jewelry shops, home etc.
\end{abstract}

\section{Keywords}

PIR Sensors, Raspberry Pi, Buzzer, Relay and GSM 900

\section{INTRODUCTION}

The proposed system is based on concept of IoT " Everything that is connected to the internet is alive", is going to be the new rule for future. Future is Internet of Things (IoT), world is moving towards it with rapid pace[9]. According to Amol Dhumane [9] objectives of IoT are:

1. To build highly interconnected system where devices will be the users of the internet.

2. This system should work 'smartly' for the betterment of human beings.

3. The system should improve the relationship between the humans and the environment in which they live.

Theft prevention would become a godsend in this increasingly technology conscious world. Many of the theft detection systems are available to catch the thief, which can be further improved. By using these technologies, in some scenarios, the thief cannot be caught. Even if the thief is caught, victim cannot get back his/her valuable belongings. "Prevention is better than cure". If the theft is being prevented from happening, the person will be at no loss.

Modern security system should be able to identify a housebreaker attempting to enter the home. After this, system should notify the victim about the invasion or any illegal activity. Also system should be able to prevent the housebreaker from entering the home as well as capturing/collecting proofs about invasion. Technology is changing day by day making the home security systems more powerful. It has changed from a simple lock and key security concept to implementing sophisticated security systems using cameras, microphones, contact sensors, proximity sensors, alarms, silent alarms, etc. The best feature about today's modern security systems is that, one can control their home devices just by using Internet.

\section{OBJECTIVES}

\section{Safety:}

When person is on vacation or belongs to corporate world theft can take place in his/her absence. This system is useful to provide security to his/her valuables. Suppose a person has kept his/her valuable antiques at home, theft of such valuable things can be prevented. Because valuable things once lost, cannot be recovered.

\section{Reliability:}

Power supply is provided by an adapter to this system; hence system is active throughout in the presence of electricity. In absence of electricity an additional battery backup is also provided to keep the system active. Hence user can rely on this system from security point of view.

\section{Evidence:}

Evidence becomes very useful parameter in any theft case. The proposed system is capable of capturing image as soon as lights are turned ON. This image is uploaded on web page with that respective time of any intruder's detection. Through this user has a strong proof in his/her hand for further investigation

\section{RELATED STUDY}

Modern security system should be able to identify a housebreaker attempting to enter the home. After this notifying the victim about the invasion or any illegal activity. Also system should be able to prevent the housebreaker from entering the home as well as capturing/collecting proofs about invasion. Technology is changing day by day making the home security systems more powerful. It has changed from a simple lock and key security concept to implementing sophisticated security systems using cameras, microphones, contact sensors, proximity sensors, alarms, silent alarms, etc. The best feature about today's modern security systems is that, one can control their home devices just by using Internet.

In some security systems, IR sensors are used to sense the presence of a human (housebreaker). Then it notifies the homeowner about the illegal activity or theft and buzzer starts ringing. The notification to user is send by Bluetooth or SMS. The user becomes aware of intrusion by receiving notification. Also people in the neighborhood will become alert of the theft because of alarm. The homeowner can take appropriate action after this.

An infrared sensor is an electronic instrument which is used to 
sense certain characteristics of its surroundings by emitting and/or detecting infrared radiation. Infrared sensors are also capable of measuring the heat being emitted by an object and detecting motion. IR sensors need to have direct line of sight between transmitter and receiver because it does not work through walls or doors. They must be almost directly aligned (i.e. able to see each other) to communicate. They are blocked by common materials such as people, walls, plants, etc. Due to short range performance drops off with longer distance. They are affected by environmental conditions like direct sunlight, rain, fog, dust and pollution. Data transmission rate is lower than wired transmission.

Bluetooth is wireless LAN technology which is designed to connect devices with different functionality for example telephone, notebook, computer (desktop, laptop), camera, printer when these devices are at short distance from each other. It is a ad-hoc network that is formed spontaneously. Maximum communication range of Bluetooth is $100 \mathrm{~m}$ in any ideal conditions. But that is not sufficient for home environment. Bluetooth communication has comparatively high power consumption, so the batteries of devices need to be frequently recharged or replaced. Bluetooth technology has advanced and improved to Bluetooth Low Energy (BTLE), which provides the same range of communication. However, it has serious security concerns such as eavesdropping and weak encryption. Hence Bluetooth becomes unreliable for sending notification to homeowner.

The security systems which use Bluetooth and IR sensor have some limitations. IR sensors can sense anything passing through it which may a living or nonliving thing. After sensing any object it notifies homeowner as well as rings the alarm. This creates problem for the user as well as the people in the neighborhood. This becomes problematic and tedious because whenever alarm rings the nearby people will reach that location for checking if there is something wrong. Also the user who is far away from that location will return back because he has received notification about invasion. This will happen every time even if any object is detected.

It will be better approach if PIR sensors are used. The PIR Sensors can detect the Infrared Rays released by human body. The light or any other electrical appliance can be activated automatically by the active presence of a human body within the detection range or coverage area \& when there is no presence the light will be deactivated automatically. Even if any object comes in its range it will not detect because it is only programmed to detect those bodies that radiates the heat. So the advantage of PIR sensor is that it will detects only living things. This feature is very beneficial in the security systems.

Consider a scenario where the homeowner is out of station for 4-5 days, and if any intruder tries to invade the home. In that case proposed system uses PIR sensors for detecting human presence. If any human presence is detected it will turn the lights $\mathrm{ON}$ with the help of relay and the buzzers stats ringing. Camera is used to capture the image of intruder immediately after the lights are turn ON. Homeowner will be notified about the intrusion through SMS. The captured image will be uploaded on a web page as evidence or proof about the intrusion. This system is more efficient and feasible because the user as well as the neighborhood will get aware about this illegal activity. The homeowner will have image as evidence of intruder to take appropriate action. The theft is prevented because after the lights are turned on and the ringing of alarm will make the intruder unsuccessful in executing his plan. The intruder would escape the location and the theft is prevented.

This system focuses on the security aspect of the existing home automation system and points out its flaws. It shows how the concept of security and meaning of the word "intruder" has changed in modern homes. The paper points out the shortcomings of existing home automation systems in identifying and preventing sophisticated intruders in a home environment. For future work in the field of home automation security, effort is to encourage the researchers to consider a home automation system as a whole and develop behavior prediction and advanced sensing parameters that can help to identify and prevent skilled and sophisticated intruders. Security is vital for the proper implementation and development of the home automation systems. Moreover, it provides a sense of security to a home's inhabitants and puts their minds at ease.

\section{COMPONENTS OF PROPOSED SYSTEM}

\subsection{Raspberry Pi:}

The system uses original Raspberry Pi 2 Model B having 40 pins. It has 26 GPIO pins, 4 USB 2.0 ports. It has a better hotplug as well as over-current behavior. It also has easy-to-use micro SD version with push-push facility. It is power-efficient by replacing linear regulators. Raspberry pi module acts as server; it receives radiations from PIR sensors as input. It then activates the relay module. When image is captured it is stored in Raspberry pi. It is also responsible for activating GSM module for notification purpose. 


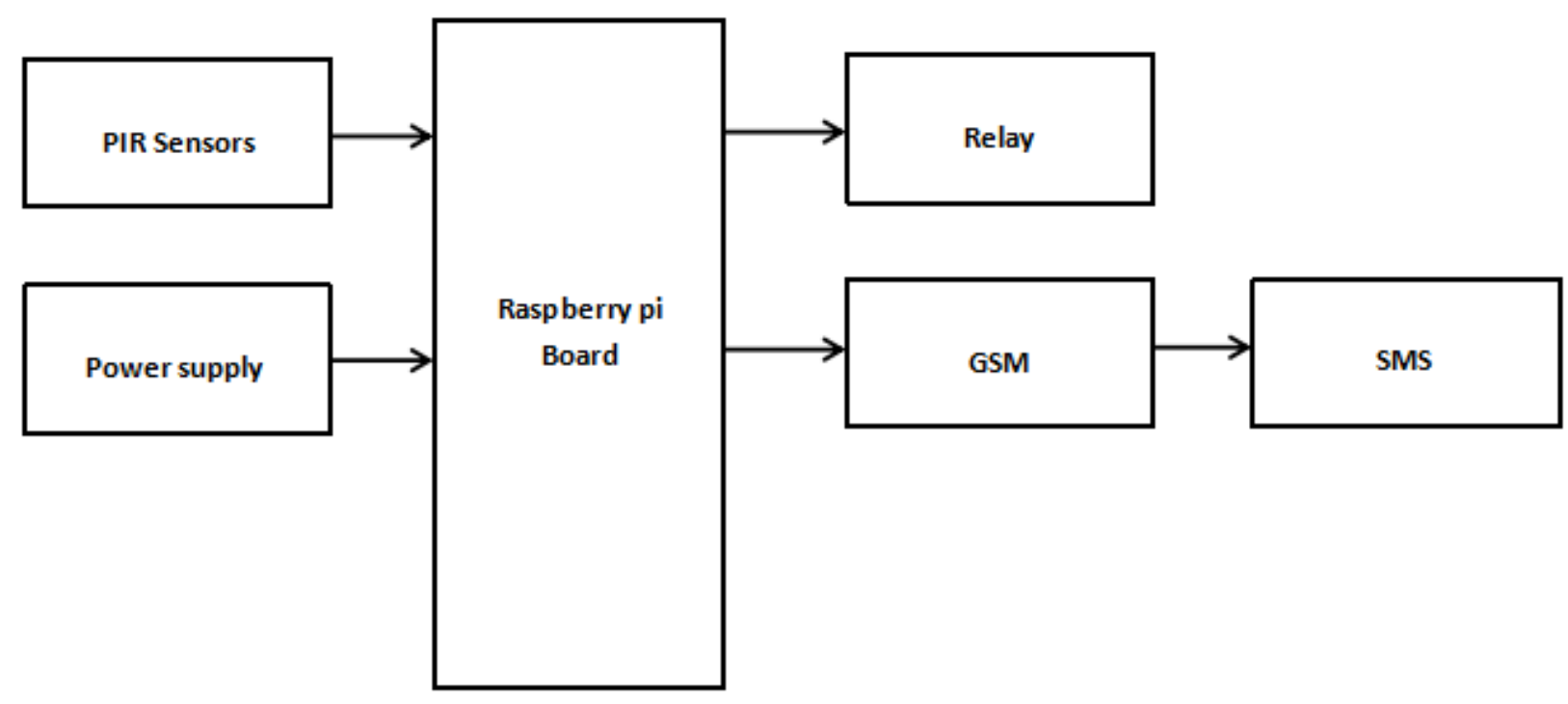

Figure 1: System Overview

\subsection{PIR Sensor}

PIR sensors stands for Passive Infrared. It is also known as motion detector. Sensitivity range of PIR sensor is up to $20 \mathrm{ft}$.(6 meters) 110 degree*70 degree .PIR sensor are used to detect infrared radiations emitted from the warm blooded bodies and they do not detect or measure heat. It is also referred as Pyroelectric or PID i.e. Passive Infrared Detector. PIR sensors are commonly useful in application where there is need of detecting presence of human in specified area.

\subsection{Relay}

Relay is a simple electro-mechanical switch. It set of contacts made up of an electromagnet. Relays are commonly used in home appliances by which one can control turning on/off of appliances like a motor, light, etc.

\subsection{GSM SIM}

GSM stands for Global Standard for Mobile communication. Different systems can use the GSM SIM to communicate over any telecommunication network. GSM is used to send and receive text message.

\section{ARCHITECTURAL FLOW}

Following Figure shows the architectural flow of system.

This system works as follows:

1. Consider a scenario where an intruder enters in a room for attempting theft.

2. If he comes in range of sensor; signals are sent to Raspberry Pi.

3. Raspberry Pi acts as server and controls relay and GSM Module.

4. Relays will lead to turn ON and OFF the lights.

5. After these buzzer will start ringing.

6. Image is captured and uploaded on web page.

7. GSM module sends notification to the victim so that he becomes aware of intrusion.

This will lead to prevention of theft.

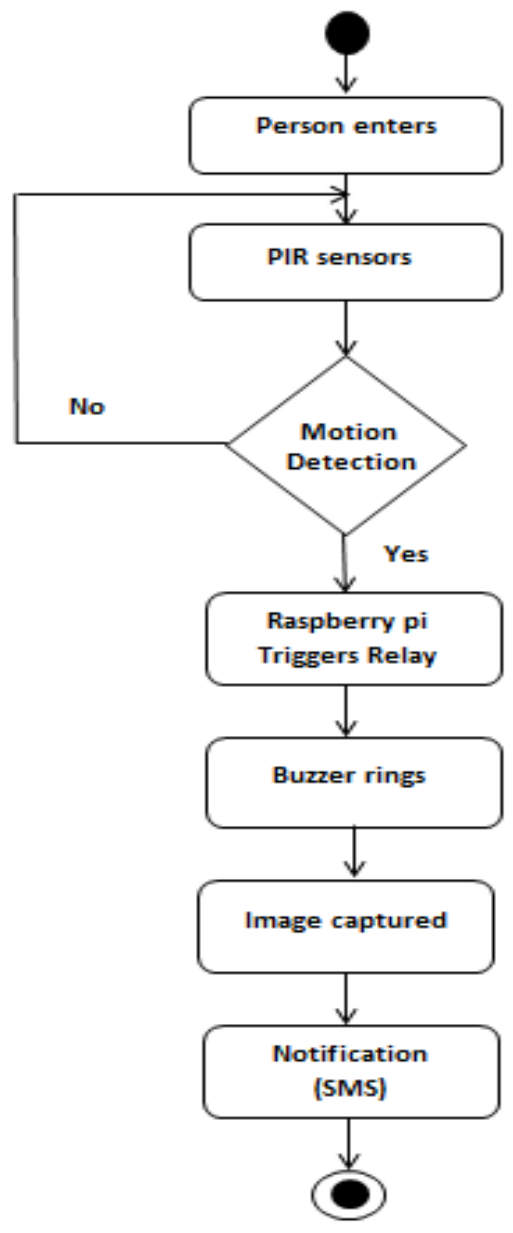

Fig 2: Flowchart of Proposed System

\section{CONCLUSION}

By referring related work different designs can be understood, architecture and implementation attempted on proposed system. The proposed system is capable of detecting presence of intruder using Raspberry Pi as server module. This will lead in prevention of theft. 


\section{FUTURE SCOPE}

From improvement point of view we can add new features to existing system such as, providing delay timer to the system so that owner can switch off the system and by using framing techniques video can also be captured as an evidence.

\section{REFERENCES}

[1] Sharma, Rupam Kumar, et al. "Android interface based GSM home security system." Issues and Challenges in Intelligent Computing Techniques (ICICT), 2014NInternational Conference on. IEEE, 2014.

[2] De Luca, Gabriele, et al. "The use of NFC and Android technologies to enable a KNX-based smart home." Software, Telecommunications and Computer Networks (Soft COM), 2013 21st International Conference on. IEEE, 2013.

[3] Gu, Yi, et al. "Design and Implementation of UPnP-Based Surveillance Camera System for Home Security." Information Science and Applications (ICISA), 2013 International Conference on. IEEE, 2013.

[4] Van Thanh Trung, Bui, and Nguyen Van Cuong. "Monitoring and controlling devices system by GPRS on FPGA platform." Advanced Technologies for
Communications (ATC), 2013 International Conference on. IEEE, 2013.

[5] Karia, Deepak, et al. "Performance analysis of ZigBee based Load Control and power monitoring system." Advances in Computing, Communications and Informatics (ICACCI), 2013 International Conference on. IEEE, 2013.

[6] Ryu, Yeonghyeon, Jeakyu Yoo, and Youngroc Kim. "Cloud services based Mobile monitoring for Photovoltaic Systems." Cloud Computing Technology and Science (Cloud Com), 2012 IEEE $4^{\text {th }}$ International Conference on. IEEE, 2012.

[7] Robson, Clyde, et al. "High performance web applications for secure system monitoring and control." Nuclear Science Symposium and Medical Imaging Conference (NSS/MIC), 2012 IEEE. IEEE, 2012.

[8] Han, Jinsoo, et al. "User-friendly home automation based on 3D virtual world." Consumer Electronics, IEEE Transactions on 56.3 (2010): 1843-1847.

[9] Amol Dhumane, Rajesh Prasad, Jayashree Prasad, "Routing Issues in Internet of Things: A Survey", Proceedings of the International Multi Conference of Engineers and Computer Scientists 2016 Vol I, IMECS 2016, March 16 - 18, 2016, Hong Kong. 